\title{
Using a Computational Model of Cardiac Fibroblast Signaling to Screen for Drugs Effective Against Heart Failure
}

\section{Anirudha S. Chandrabhatla, Angela C. Zeigler, Jeffrey J. Saucerman}

\section{Abstract}

Cardiac fibroblasts are the cells responsible for remodeling the heart's extracellular matrix (ECM) after a myocardial infarction. ECM breakdown during the early, inflammatory phase of remodeling should be balanced with deposition of new collagen during the proliferative phase in order to maintain cardiac function and prevent heart failure. Currently, it is not clear which drug(s) would best modulate ECM production by fibroblasts because relevant proteins and signaling pathways are typically studied in isolation. In this study, we adopted a systems biology approach to perform a comprehensive drug screen using a fibroblast signaling network that integrated 10 signaling pathways with 104 molecules and 157 reactions. We developed a differential equation model for the reactions in the network and used the DrugBank database to obtain information on 114 FDA approved/investigational drugs that had a target within our network. We simulated the addition of each drug to the model in either the proliferative or inflammatory phases and observed the foldchange in activity of 8 network outputs, including collagen I and III protein. Drugs such as Arbutamine, Amyl Nitrate and Arsenic Trioxide were predicted to decrease collagen levels during the proliferative phase, thereby potentially helping prevent excess ECM deposition. Siltuximab and Triflusal were predicted to increase collagen protein during the inflammatory phase, thereby working to prevent excess ECM degradation. 


\section{Introduction}

Fibroblasts are the cells responsible for producing structural proteins that form the extracellular matrix (ECM) (Fan et al., 2012). In the heart, fibroblasts account for two-thirds of all cells and contribute to myocardial stability, coordinate responses to mechanical stimuli and help remodel tissue after cardiac injury (MacKenna et al., 2000).

One of the most common examples of cardiac injury is an acute myocardial infarction (MI). Cardiomyocyte death from an MI triggers a pro-inflammatory cellular environment that induces the migration of leukocytes, macrophages and fibroblasts. This phase of healing post-MI, deemed the "inflammatory phase", is characterized by ECM degradation by fibroblasts and the subsequent clearing of dead cells by macrophages. As dead cells are cleared out of the infarct, secretion of signaling molecules such as Transforming Growth Factor Beta (TGF $\beta$ ) marks the end of the inflammatory phase and beginning of the "proliferative phase". Since adult cardiomyocytes are terminally differentiated, there is no possibility of new heart cells growing to replace the dead cells. Therefore, in the proliferative phase, fibroblasts themselves proliferate and deposit collagen in the infarct (Dobaczewski et al., 2010).

The wound healing process post-MI is tightly controlled, in that ECM breakdown during the inflammatory phase must be balanced with deposition of collagen in the proliferative phase. Pathological remodeling with excessive ECM breakdown and insufficient collagen deposition can lead to cardiac dilation. On the other hand, insufficient ECM breakdown and excessive collagen deposition can lead to cardiac fibrosis. Both dilation and fibrosis eventually lead to heart failure, which currently affects 
over 23 million people worldwide and costs the United States healthcare system \$32 billion dollars a year (Bui et al., 2011; Mozaffarian et al., 2015). Since fibroblasts are the cells responsible for remodeling the ECM, it would be ideal to therapeutically target their activity to prevent heart failure.

Currently, it is not clear what drug(s) would best modulate production of ECM by fibroblasts because relevant proteins or signaling pathways are typically studied in isolation. Previous studies have examined the effects of disrupted IL-1 signaling on cardiac remodeling (Bujak et al., 2008) or have shown that Angiotensin II Receptor inhibitors decrease collagen production and secretion in fibroblasts (Lijnen et al., 2000). However, there have not been studies that combine multiple fibroblast signaling pathways in order to examine gene expression and protein activity across the inflammatory and proliferative phases. In this study, we adopted a systems biology approach to perform a comprehensive, mechanistic drug screen using an experimentally validated fibroblast signaling network. The overall goal of the study was to identify drugs that could control/modulate cardiac fibroblast activity and prevent the development of heart failure.

Drugs likely affect the heart differently in the inflammatory versus the proliferative phases. Our computational model allowed us to simulate those stages of wound healing while also enabling us to examine the effects of different drug properties. Specifically, we were interested in studying the effects of competitive and non-competitive binding, since it has been shown in the literature that drug effects vary depending on whether the drug bound its target competitively or non-competitively (Jensen et al., 2016). 
Here we developed a methodology that utilizes a cardiac fibroblast signaling network to computationally screen for drugs that could prevent excess ECM breakdown or deposition. In both the competitive and non-competitive drug simulations, we identified Siltuximab and Triflusal as drugs that increased activation of Collagen I (Cl) and Collagen III (CIII) and Arbutamine, Amyl Nitrate and Arsenic Trioxide as drugs that decreased activation of both collagens. We also observed differential effects of Arbutamine and Amyl Nitrate on $\mathrm{Cl} \& \mathrm{CIII}$ based on whether the drugs were simulated as competitive or non-competitive.

\section{Methods}

\section{Fibroblast Signaling Network}

The cardiac fibroblast signaling network was constructed using data from previous experimental studies in the literature. The network incorporated 104 nodes (mRNA, proteins and cell processes) connected by 157 reactions and was converted into a logic-based ordinary differential equation (ODE) computational model as described in previous studies (Kraeutler et al., 2010; Ryall et al., 2012; Zeigler et al., 2016). The activity of each node was modeled using a normalized Hill ODE with default reaction parameters of weight (1), Hill coefficient (1.4) and EC50 (0.6). Each node had $y_{\text {init }}(0), y_{\max }(1)$ and a time constant, $\tau$, that was scaled based on the type of reaction. The network was developed using 84 papers and 34 papers were used to validate the prediction of node activity. Overall, the network correctly validated 66 of 82 comparisons (80\%). 


\section{DrugBank}

DrugBank provided a .csv file of 8207 FDA-approved and investigational drugs.

Using BeautifulSoup 3.0, a Python

HTML/XML parsing library, we

data-mined the DrugBank website

(Law et al., 2014) to extract more

information such as the drug target

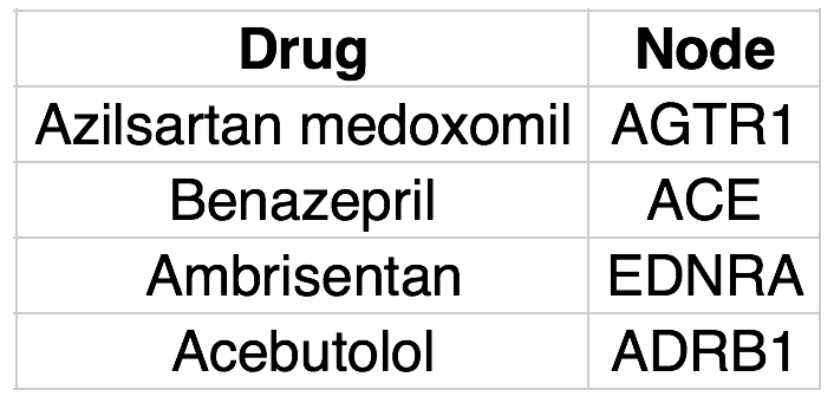

Figure 1. Four of the 30 unique drug-node pairings.

and type of drug. In MATLAB, we compared the GeneCard IDs of the nodes in our network to those of the drug targets to generate a list of 114 drug-node pairs. The drugs were further classified as antagonists or agonists to generate a list of 30 unique drug-node pairs, a subset of which is shown in Figure 1.

\section{Simulations}

The system of ODEs underlying our fibroblast signaling network was generated using the Netflux software available at: https://github.com/saucermanlab/Netflux. Netflux provided MATLAB files for the model parameters (Weight, Hill Coefficient, etc.) of each reaction in the network, the ODE model itself and the initial activation of all the nodes.

The extracellular environments during the inflammatory and proliferative phases were gleaned from the literature (Bujak et al., 2008; Dewald et al., 2004; White et al., 2001). Specifically, the inflammatory phase was characterized by increased activation of IL1, IL6 and Tumor Necrosis Factor Alpha (TNF $\alpha$ ) while the proliferative phase was characterized by increased activation of Angiotensin II (ANGII), TGF $\beta$, Norepinephrine (NE), Endothelin 1 (ET1) and Natriuretic Peptide (NP). The upregulation of these 
signaling molecules was simulated by increasing the weight of the appropriate input reactions. The weights of all input reactions in the model were set to a default value of 0.25. To simulate the inflammatory phase, for example, the weights for IL1, IL6 and TNF $\alpha$ input were increased to 0.5 .

Un-perturbed simulations, without the addition of any drugs, were run using MATLAB ODE15s to establish baseline activation in the wound healing phase being simulated. We ran four perturbed simulations for each drug: 1) Inflammatory phase, simulating the drug as competitive, 2) Inflammatory, drug as non-competitive, 3)

Proliferative, drug as competitive, 4) Proliferative, drug as non-competitive.

All simulations took the type of drug into account: agonist or antagonist. Briefly, agonists increase activity of their targets while antagonists decrease activity. We also took the drugs' mechanisms - either competitive or non-competitive - into consideration. Figure 2 shows the effect of simulating the addition of an antagonist that acts on a simple, $\mathbf{A} \rightarrow \mathbf{B}$ reaction. The blue curve depicts the activation of $B$ without the addition of a drug. The orange curve shows the characteristic response after the addition of a competitive antagonist in which there

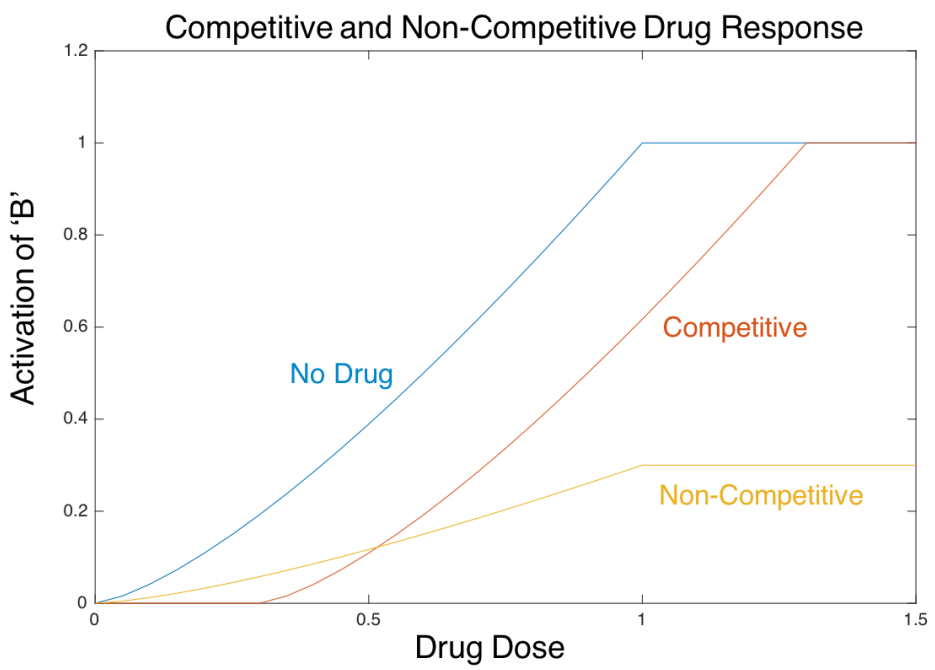

Figure 2. The responses to a competitive and non-competitive antagonist. The competitive curve shifts to the right, changing the EC50 but not the maximal activation. The non-competitive curve is scaled down, changing the maximal activation but not the EC50.

is a change in the EC50 - the drug concentration required to achieve half maximal 
response - but not the maximal activation. The yellow curve depicts the characteristic response after the addition of a non-competitive antagonist in which there is a change in maximal activation but not in the EC50.

The Hill Equation used to calculate activation of the nodes was: $f_{a c t}(X)=w \frac{\beta X^{n}}{K^{n}+X^{n}}$ ' $\beta$ ' and ' $K$ ' were parameters used to constrain the function such that $f_{a c t}(0)=0$, $f_{a c t}(E C 50)=0.5$ and $f_{a c t}(1)=1$. ' $X$ ' is the activation of the node directly upstream, ' $w$ ' is the weight of the reaction and ' $n$ ' is the Hill Coefficient (set to 1.4 in our model).

We ran non-competitive simulations by altering the weight(s) of the reaction(s) in which the drug target was a product. For example, the drug Benazepril is an antagonist that targets Angiotensin Converting Enzyme (ACE). There was only one reaction in our network that fed into ACE (TGFB1R $\rightarrow$ ACE), so when simulating the addition of Benazepril, we decreased the weight of that reaction to $50 \%$ of its default value. For agonists, we increased the weights to $150 \%$ of their default values. The weights could be scaled differently to model different doses.

We ran competitive simulations by altering ' $\mathrm{X}$ ', the activity of a node's upstream regulator(s). Specifically, in order to achieve the shift in EC50 without changing the maximal activation, the Hill Equation became $f_{a c t}(X-a)=w \frac{\beta(X-a)^{n}}{K^{n}+(X-a)^{n}}$ where 'a' was positive for agonists and negative for antagonists.

Simulations without drugs were run to steady state and afterwards, we ran the simulations with competitive or non-competitive drugs. The steady state activations of all nodes were recorded, paying special attention to Alpha Smooth Muscle Actin (aSMA), Matrix Metalloproteinases (MMP) 1, $2 \&$ 9, Cl \& CIII protein, and Cl \& CIII 
mRNA. The foldchange was calculated by dividing the steady state, un-perturbed activation by the steady state, perturbed activation.

\section{Results}

\section{Non-Competitive Simulations}

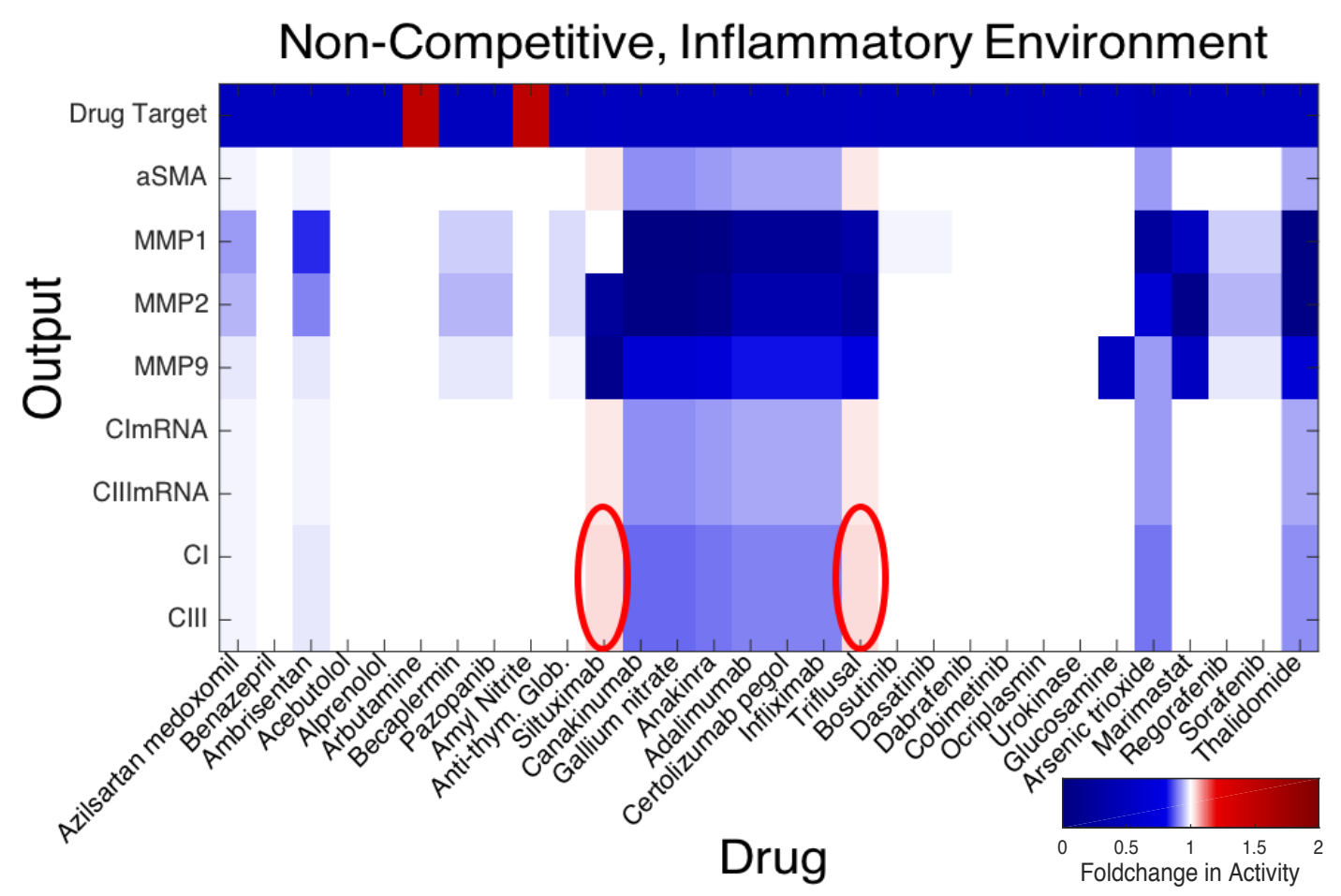

Figure 3. The effect of simulating the 30 drugs on 8 selected outputs of the signaling network during the inflammatory phase post-MI. All drugs were modeled as noncompetitive agonists or antagonists. The circled areas show that Siltuximab and Triflusal increased $\mathrm{Cl}$ and Cll activation.

The heatmap seen in Figure 3 depicts the effects on 8 selected outputs of the network when the 30 drugs were modeled non-competitively in the inflammatory environment. Siltuximab and Triflusal were considered promising drugs in this phase of wound healing because they would increase $\mathrm{Cl} \& \mathrm{CIII}$ levels and help prevent excess ECM degradation. 


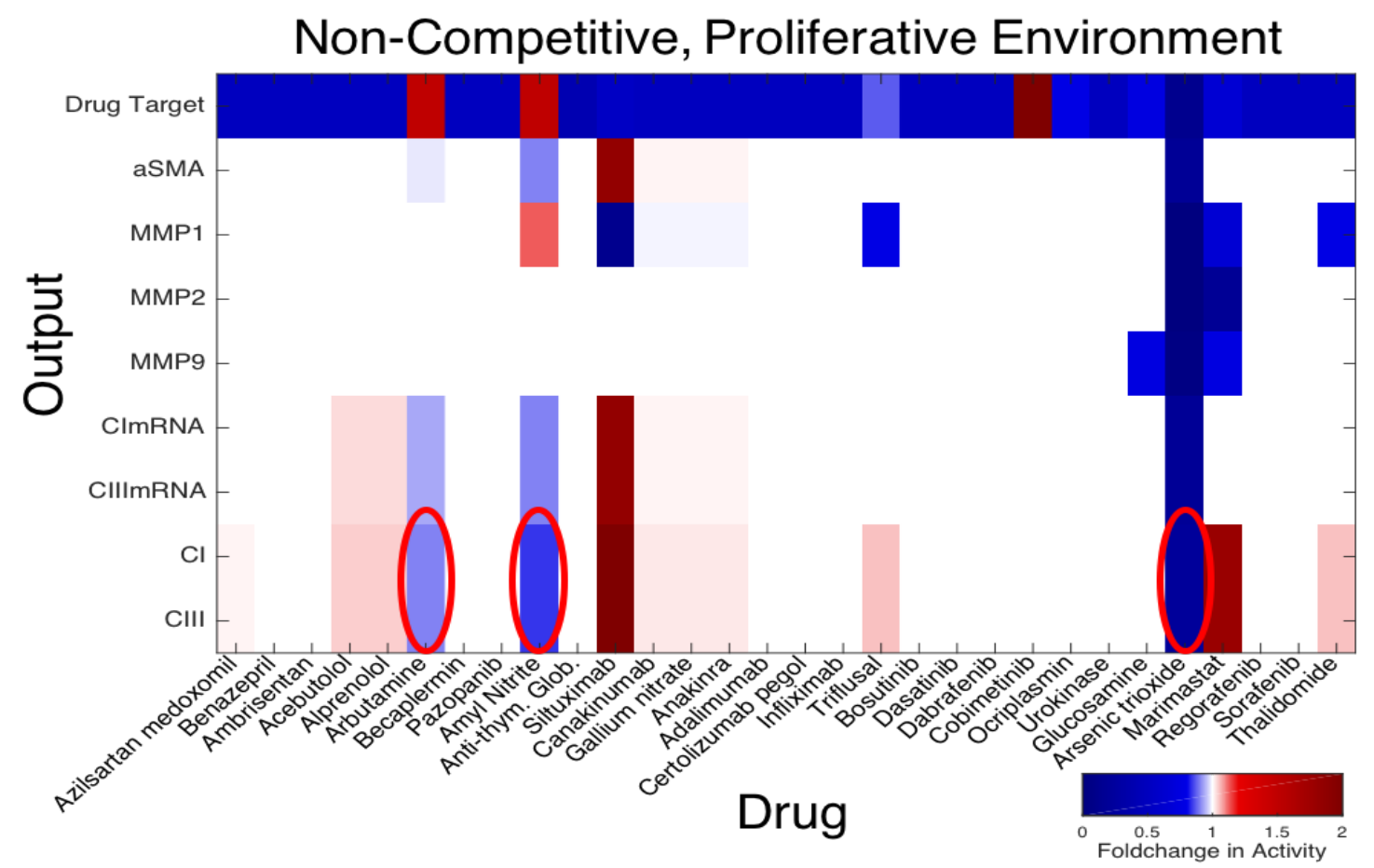

Figure 4. The effect of simulating the 30 drugs on 8 selected outputs of the signaling network during the proliferative phase post-MI. All drugs were modeled as noncompetitive agonists or antagonists. The circled areas show that Arbutamine, Amyl Nitrate and Arsenic Trioxide decreased $\mathrm{Cl}$ and Cll activation.

The non-competitive simulations were re-run for the proliferative phase post-MI.

Arbutamine, Amyl Nitrate and Arsenic Trioxide were considered promising drugs in this phase of wound healing because they would decrease CI \& CIII levels and help prevent excess ECM deposition (Figure 4). 


\section{Competitive Simulations}

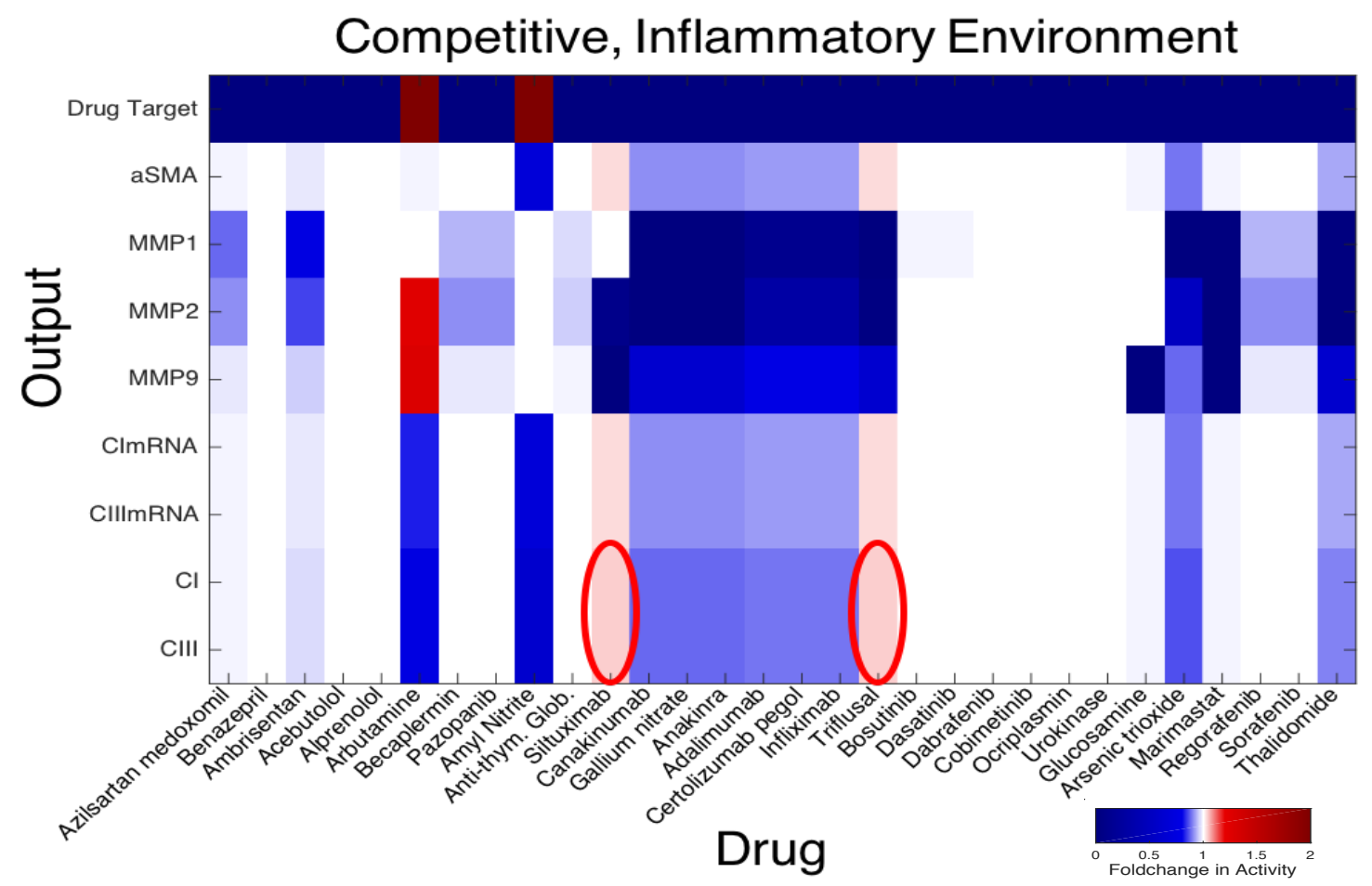

Figure 5. The effect of simulating the 30 drugs on 8 selected outputs of the signaling network during the inflammatory phase post-MI. All drugs were modeled as competitive agonists or antagonists. The circled areas show that Siltuximab and Triflusal increased $\mathrm{Cl}$ and Cll activation.

We simulated the same drugs in the same environments, this time modeling the drugs as competitive agonists/antagonists. As seen with the non-competitive, inflammatory simulations, Silutimab and Triflusal were the promising drugs that increased $\mathrm{Cl} \& \mathrm{CIII}$ levels (Figure 5). 


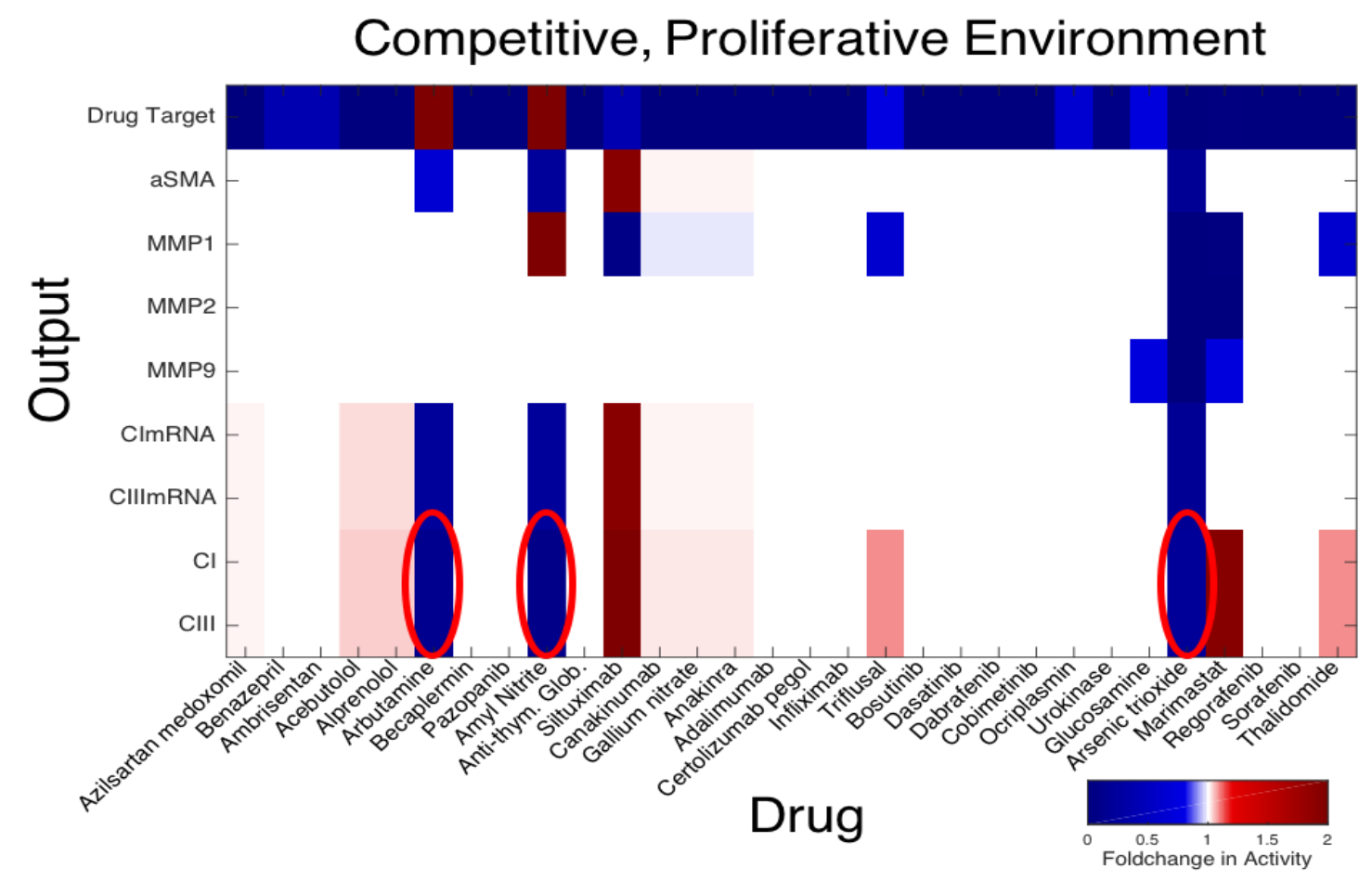

Figure 6. The effect of simulating the 30 drugs on 8 selected outputs of the signaling network during the proliferative phase post-MI. All drugs were modeled as competitive agonists or antagonists. The circled areas show that Arbutamine, Amyl Nitrate and Arsenic Trioxide decreased $\mathrm{Cl}$ and Cll activation.

Arbutamine, Amyl Nitrate and Arsenic Trioxide continued to be the promising drugs in the proliferative phase of wound healing. However, the competitive simulations show an increase in the effect the three drugs had on $\mathrm{Cl} \& \mathrm{CIII}$, as seen by the darker blue bars in Figure 6. 


\section{Effects of Promising Drugs on Cl/CII}

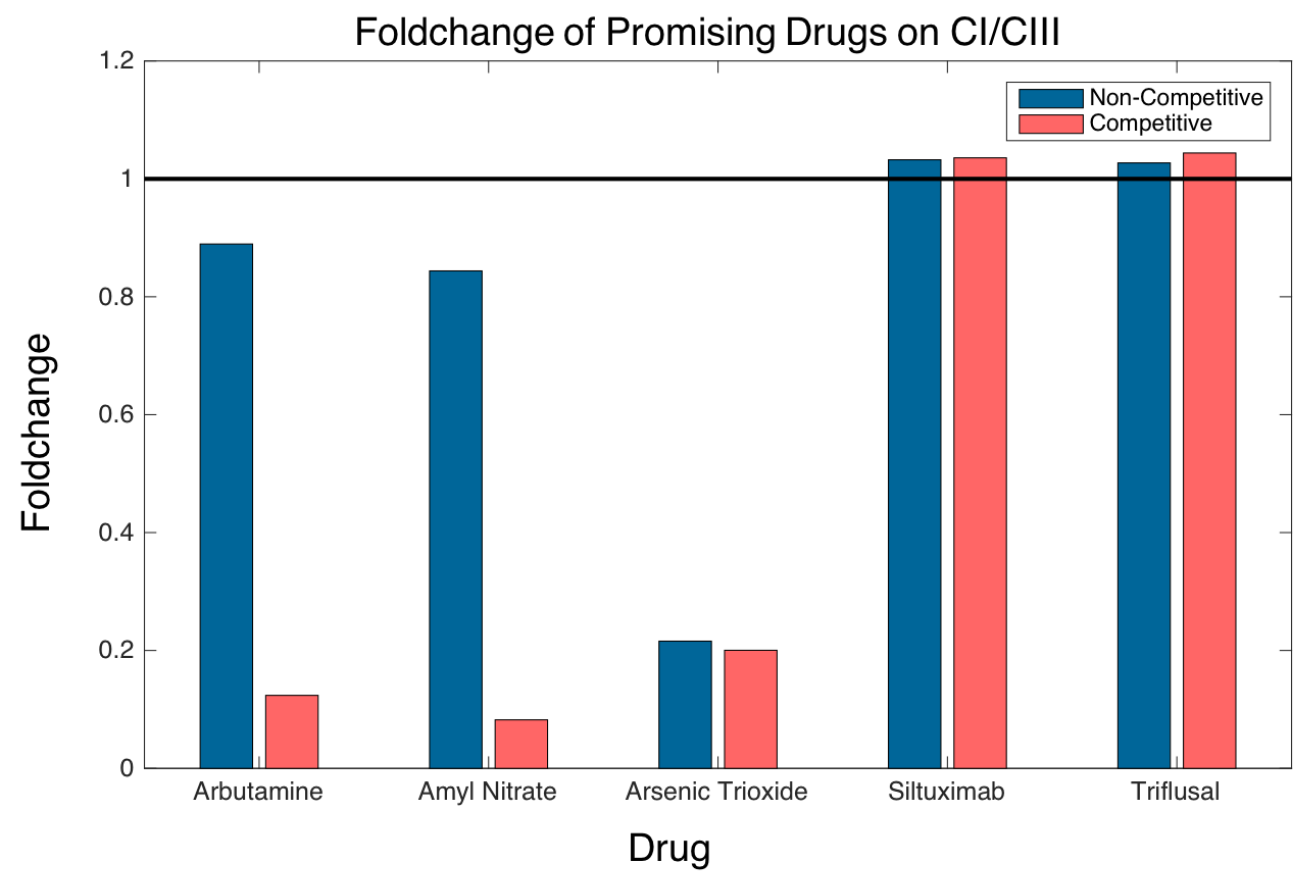

Figure 7. The foldchange effect of the 3 promising drugs in the proliferative phase (Arbutamine, Amyl Nitrate \& Arsenic Trioxide) and 2 from the inflammatory phase (Siltuximab \& Triflusal) across both the competitive and non-competitive simulations. The black, horizontal line indicates no change in activity. The effect of Arbutamine and Amyl Nitrate was more pronounced in the competitive compared to the non-competitive simulations.

Figure 7 summarizes the effects of the five promising drugs on $\mathrm{Cl} \& \mathrm{CIII}$. The data for Arbutamine, Amyl Nitrate and Arsenic Trioxide were from the proliferative simulations while the data for Siltuximab and Triflusal were from the inflammatory simulations. All drugs affected $\mathrm{Cl}$ and $\mathrm{Clll}$ equally, resulting in the same foldchange values for both proteins. Siltuximab, with foldchange effects of 1.0357 and 1.0324 for the competitive and non-competitive simulations respectively and Triflusal, with foldchange effects of 1.0439 and 1.0271 , were barely above a foldchange of 1 , which would indicate no effect at all.

Arbutamine and Amyl Nitrate displayed the largest change in activity between the competitive and non-competitive simulations. Arbutamine's effects on $\mathrm{Cl} / \mathrm{Clll}$ increased 
7 -fold in the competitive simulations ( 0.1283 non-competitive to 0.8895 competitive) and Amyl Nitrate's effects increased 10 -fold (0.0823 to 0.8439$)$.

\section{Altered Effects in Competitive and Non-Competitive Simulations}
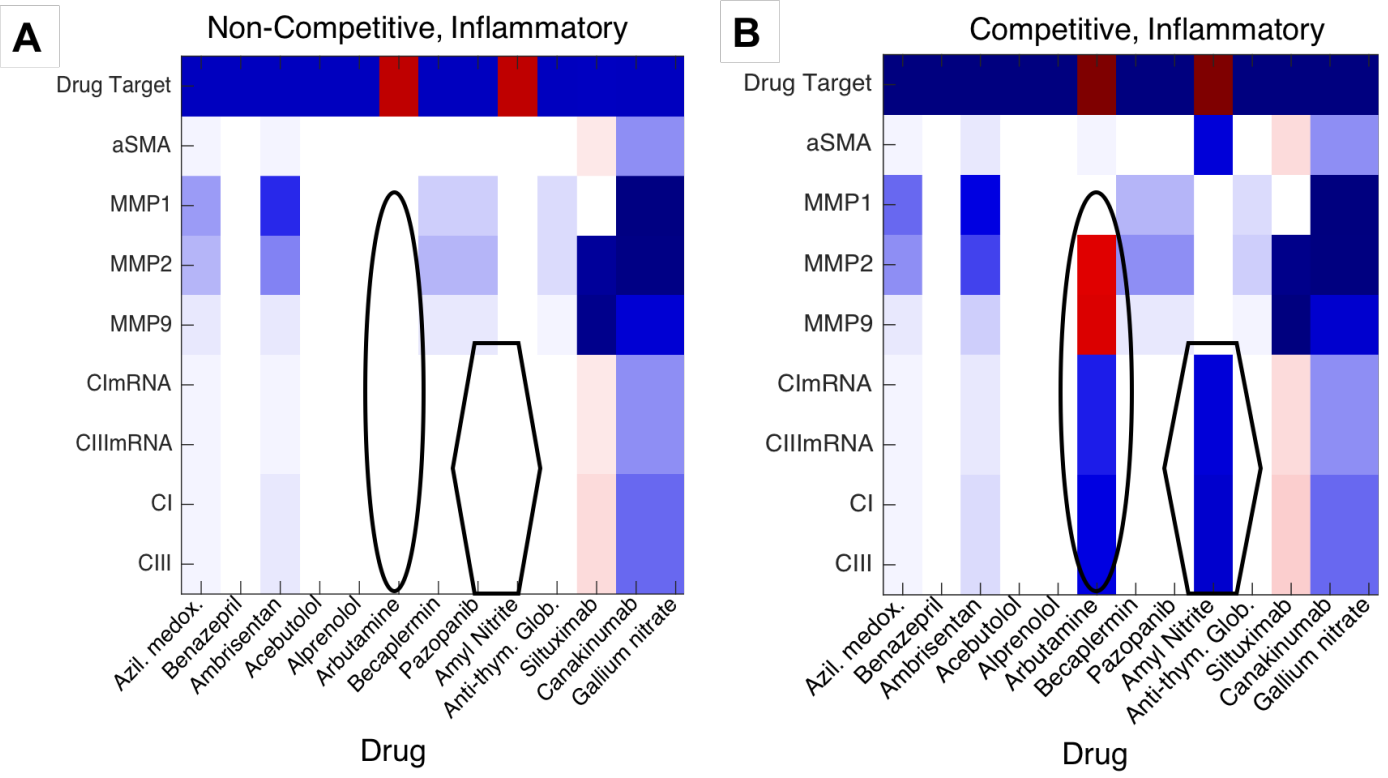

Figure 8. Drug effects were dependent on whether the drugs were simulated as non-competitive (A) or competitive (B).

As discussed in Jensen et al., we observed changes in drug effects based on whether the drugs were simulated as competitive or non-competitive. Specifically, Arbutamine and Amyl Nitrate had no effects on $\mathrm{Cl}$ or $\mathrm{Clll}$ when simulated as noncompetitive agonists, but decreased both proteins' activities when simulated as competitive agonists (Figure 7). 


\section{Discussion}

In this study, we developed a methodology that utilized a cardiac fibroblast signaling network to computationally screen for drugs that could prevent excess ECM breakdown or deposition. We compared drug action in two different wound healing environments and examined how drug mechanisms (Competitive and Non-Competitive) altered the drugs' effects. In both the competitive and non-competitive simulations, Siltuximab and Triflusal were found to prevent excess ECM breakdown while Arbutamine, Amyl Nitrate and Arsenic Trioxide could prevent excess ECM deposition. We also observed differential effects of Amyl Nitrate and Arbutamine on $\mathrm{Cl} \& \mathrm{Clll}$ based on whether the drugs were simulated as competitive or non-competitive. Specifically, the two drugs had a greater effect on collagen if modeled as competitive.

In their 2016 paper reviewing the status quo of research for the treatment of myocardial fibrosis, Gyöngyösi et al. discussed the importance of high throughput screening (HTS) to allow for the testing of thousands of drugs and the subsequent measurement of multiple biological parameters to determine the drugs' efficacies (Gyöngyösi et al., 2017). Many studies have developed techniques to conduct these types of screens as recently as Kithcart et al., who used Zebrafish embryos for experimental HTS of cardiovascular drugs (Kithcart and MacRae, 2017). However, Gyöngyösi et al. also stress the importance of leveraging the increasing power of computers to conduct purely computational drug screens. Our study is the first in its field to use a computational model of fibroblast signaling to identify drugs that have the potential to prevent dilation and fibrosis. We developed a methodology to both identify 
drugs of interest from a database and run simulations of adding those drugs to our network. Our drug simulations were able to reflect, to some degree, the complexity of drug-target interactions by accounting for the type of drug and type of drug binding. At its core, this project resulted in the identification of drugs that could prevent excess ECM breakdown or deposition, which can now be tested using in vitro models.

Luo et al. showed Arsenic Trioxide to be anti-fibrotic by studying its inhibition of bleomycin-induced pulmonary fibrosis (Luo et al., 2014). Although the Luo study aligns with our simulations of Arsenic Trioxide, many of the promising drugs we identified have not been studied for their cardio-protective effects. However, the results of our simulations could lead to studies that repurpose drugs for use in post-MI treatment. For example, Siltuximab, which was approved by the FDA in 2014 to treat Castleman's disease (Barquero, 2015), is currently being studied as a potential chemotherapy drug (Chen and Chen, 2015; Hunsucker et al., 2011) and, based on the results of our work, could be studied as a collagen enhancing drug as well.

One of the limitations of our signaling network is its complete focus on fibroblasts. Other cell types, such as macrophages, play a critical role in collagen degradation and synthesis post-MI. Studies such as Yano et al. showed that injecting macrophage colony-stimulating factor into rats that recently had an $\mathrm{MI}$ increased the levels of $\mathrm{Cl}$ and $\mathrm{CIII}$ in the heart (Yano et al., 2006).

Future work will involve expanding the network to include other proteins and signaling molecules involved in collagen production/deposition. It is likely that computational modeling's role in drug screening will expand in the coming years. 


\section{Acknowledgements}

This work was funded by the University of Virginia Center for Undergraduate Excellence "Double-Hoo" grant. Special thanks to Angela Zeigler and Jeff Saucerman for their encouragement and support.

\section{Bibliography}

Barquero, N. (2015). Siltuximab: a new option for the management of Castleman's disease. Drugs Today Barc. Spain 1998 51, 21-28.

Bui, A.L., Horwich, T.B., and Fonarow, G.C. (2011). Epidemiology and risk profile of heart failure. Nat. Rev. Cardiol. 8, 30-41.

Bujak, M., Dobaczewski, M., Chatila, K., Mendoza, L.H., Li, N., Reddy, A., and Frangogiannis, N.G. (2008). Interleukin-1 Receptor Type I Signaling Critically Regulates Infarct Healing and Cardiac Remodeling. Am. J. Pathol. 173, 57-67.

Chen, R., and Chen, B. (2015). Siltuximab (CNTO 328): a promising option for human malignancies. Drug Des. Devel. Ther. 9, 3455-3458.

Dewald, O., Ren, G., Duerr, G.D., Zoerlein, M., Klemm, C., Gersch, C., Tincey, S., Michael, L.H., Entman, M.L., and Frangogiannis, N.G. (2004). Of mice and dogs: species-specific differences in the inflammatory response following myocardial infarction. Am. J. Pathol. 164, 665-677.

Dobaczewski, M., Gonzalez-Quesada, C., and Frangogiannis, N.G. (2010). The extracellular matrix as a modulator of the inflammatory and reparative response following myocardial infarction. J. Mol. Cell. Cardiol. 48, 504-511.

Fan, D., Takawale, A., Lee, J., and Kassiri, Z. (2012). Cardiac fibroblasts, fibrosis and extracellular matrix remodeling in heart disease. Fibrogenesis Tissue Repair 5, 15.

Gyöngyösi, M., Winkler, J., Ramos, I., Do, Q., Firat, H., McDonald, K., González, A., Thum, T., Díez, J., Jaisser, F., et al. (2017). Myocardial fibrosis: biomedical research from bench to bedside. Eur. J. Heart Fail. 19, 177-191.

Hunsucker, S.A., Magarotto, V., Kuhn, D.J., Kornblau, S.M., Wang, M., Weber, D.M., Thomas, S.K., Shah, J.J., Voorhees, P.M., Xie, H., et al. (2011). Blockade of interleukin6 signalling with siltuximab enhances melphalan cytotoxicity in preclinical models of multiple myeloma. Br. J. Haematol. 152, 579-592. 
Jensen, K.J., Moyer, C.B., and Janes, K.A. (2016). Network Architecture Predisposes an Enzyme to Either Pharmacologic or Genetic Targeting. Cell Syst. 2, 112-121.

Kithcart, A., and MacRae, C.A. (2017). Using Zebrafish for High-Throughput Screening of Novel Cardiovascular Drugs. JACC Basic Transl. Sci. 2, 1-12.

Kraeutler, M.J., Soltis, A.R., and Saucerman, J.J. (2010). Modeling cardiac $\beta$-adrenergic signaling with normalized-Hill differential equations: comparison with a biochemical model. BMC Syst. Biol. 4, 157.

Law, V., Knox, C., Djoumbou, Y., Jewison, T., Guo, A.C., Liu, Y., Maciejewski, A., Arndt, D., Wilson, M., Neveu, V., et al. (2014). DrugBank 4.0: shedding new light on drug metabolism. Nucleic Acids Res. 42, D1091-1097.

Lijnen, P.J., Petrov, V.V., and Fagard, R.H. (2000). Induction of cardiac fibrosis by angiotensin II. Methods Find. Exp. Clin. Pharmacol. 22, 709-723.

Luo, F., Zhuang, Y., Sides, M.D., Sanchez, C.G., Shan, B., White, E.S., and Lasky, J.A. (2014). Arsenic trioxide inhibits transforming growth factor- $\beta 1$-induced fibroblast to myofibroblast differentiation in vitro and bleomycin induced lung fibrosis in vivo. Respir. Res. 15, 51.

MacKenna, D., Summerour, S.R., and Villarreal, F.J. (2000). Role of mechanical factors in modulating cardiac fibroblast function and extracellular matrix synthesis. Cardiovasc. Res. 46, 257-263.

Mozaffarian, D., Benjamin, E.J., Go, A.S., Arnett, D.K., Blaha, M.J., Cushman, M., Das, S.R., Ferranti, S. de, Després, J.-P., Fullerton, H.J., et al. (2015). Heart Disease and Stroke Statistics-2016 Update. Circulation CIR.0000000000000350.

Ryall, K.A., Holland, D.O., Delaney, K.A., Kraeutler, M.J., Parker, A.J., and Saucerman, J.J. (2012). Network reconstruction and systems analysis of cardiac myocyte hypertrophy signaling. J. Biol. Chem. 287, 42259-42268.

White, M., Rouleau, J.L., Hall, C., Arnold, M., Harel, F., Sirois, P., Greaves, S., Solomon, S., Ajani, U., Glynn, R., et al. (2001). Changes in vasoconstrictive hormones, natriuretic peptides, and left ventricular remodeling soon after anterior myocardial infarction. Am. Heart J. 142, 1056-1064.

Yano, T., Miura, T., Whittaker, P., Miki, T., Sakamoto, J., Nakamura, Y., Ichikawa, Y., Ikeda, Y., Kobayashi, H., Ohori, K., et al. (2006). Macrophage colony-stimulating factor treatment after myocardial infarction attenuates left ventricular dysfunction by accelerating infarct repair. J. Am. Coll. Cardiol. 47, 626-634. 
Zeigler, A.C., Richardson, W.J., Holmes, J.W., and Saucerman, J.J. (2016).

Computational modeling of cardiac fibroblasts and fibrosis. J. Mol. Cell. Cardiol. 93, 7383. 